\title{
Nanostructures at surfaces from substrate-mediated interactions
}

\author{
Kristen A. Fichthorn and Michael L. Merrick \\ Department of Chemical Engineering, The Pennsylvania State University, University Park, Pennsylvania 16802, USA \\ Matthias Scheffler \\ Fritz-Haber-Institut der Max-Planck-Gesellschaft, Faradayweg 4-6, D-14195 Berlin, Germany
}

(Received 23 May 2003; published 28 July 2003)

\begin{abstract}
Recent theoretical and experimental studies indicate that adsorbate-adsorbate interactions mediated by a solid surface can be significant enough to influence the formation of nanostructures during thin-film epitaxy. Here, we show that these electronic interactions lead to the formation of repulsive barriers surrounding small adsorbate islands at surfaces. The dependence of these barriers on island size and shape actuates sharp islandsize distributions, which can be manipulated by changing growth conditions to yield selected island sizes and shapes. The existence of these interactions opens new prospects for engineering nanostructures at surfaces.
\end{abstract}

DOI: 10.1103/PhysRevB.68.041404

PACS number(s): 68.55.Ac, 81.10.Aj, 81.15.Aa

Achieving a detailed understanding of the nature and consequences of adsorbate-adsorbate interactions at solid surfaces is important in engineering properties of interfacial materials. Recent theoretical ${ }^{1-7}$ and experimental ${ }^{7-9}$ studies show that indirect interactions, mediated by the substrate, can be significant enough to influence the formation of nanostructures at surfaces. The way that these interactions influence adsorbate island formation is not understood and is the subject of this paper.

Indirect interactions can be electronic or elastic in origin. In both cases, asymptotic theories ${ }^{3,10,11}$ describe them if the separation between adatoms is sufficiently large. Much progress has been made recently in understanding adatompair interactions mediated by Shockley surface-state electrons. This oscillatory interaction decays with adsorbate separation $d$ as $d^{-2}$ and has a period related to the Fermi wave vector, as originally predicted by Lau and Kohn, ${ }^{10}$ and recently refined by Hyldgaard and Persson. ${ }^{3}$ Noble-metal fcc(111) surfaces possess these surface states and lowtemperature studies with scanning-tunneling microscopy (STM) corroborate this theory ${ }^{3}$ for adsorbate separations $>20 \AA$ on $\mathrm{Cu}(111)$ (Refs. 8 and 9) and >30 $\AA$ on $\mathrm{Ag}(111) .{ }^{9}$ At these asymptotically large separations, indirect interactions are weak (less than $2 \mathrm{meV}$ ) and inconsequential for surface morphology under typical growth conditions. However large-scale, density-functional theory (DFT) calculations ${ }^{1}$ show that these interactions can be much stronger at "intermediate" separations (i.e., separations too long for adsorbates to form direct chemical bonds and too short for the asymptotic regime).

The significance of the predicted "intermediate-range" interactions was confirmed by recent STM experiments on $\mathrm{Cu}(111),{ }^{8,9}$ where Repp et al. and co-workers observed an extra repulsive barrier of $15 \mathrm{meV}$ that prevents two $\mathrm{Cu}$ atoms from coming closer than $\sim 10 \AA .{ }^{8}$ Knorr et al. found a similar barrier in their STM study. ${ }^{9}$ Because they are repulsive, these interactions inhibit island nucleation in thin-film epitaxy, as predicted by Fichthorn and Scheffler. ${ }^{1}$ Kinetic Monte Carlo simulations s,2,4-6 $^{1,2}$ and subsequently derived mean-field theories ${ }^{5,7}$ show that this leads to higher island densities than those expected from standard nucleation theory. Experimen- tal work supports these predictions. ${ }^{9,12,13}$ In this Rapid Communication, we report on another important ramification of "intermediate-range," substrate-mediated interactions: their influence on the sizes and shapes of adsorbate islands that develop during thin-film epitaxy.

We examine the growth of Ag on compressively strained $\mathrm{Ag}(111)$ and $\mathrm{Cu}$ on $\mathrm{Cu}(111)$. Both surfaces possess intermediate-range interactions significant enough to influence the island density in thin-film epitaxy. ${ }^{1,7,12}$ In a previous study, ${ }^{1}$ we obtained the electronic pair interaction between $\mathrm{Ag}$ atoms on strained $\mathrm{Ag}(111)$ using DFT calculations. Briefly, these calculations employ the plane-wave, pseudopotential method, as implemented in the FHI98MD code, ${ }^{14}$ within the generalized-gradient approximation ${ }^{15}$ using a $(4 \times 4$ $\times 4$ ) unit cell with four $\mathbf{k}_{\|}$points in the surface Brillouin zone. This potential is shown in Fig. 1(a), where we see that the main features are a short-range attraction associated with direct chemical bonding and an "intermediate-range" repulsive ring (yellow in Fig. 1) at the 10th-13th neighbor distances.

The $\mathrm{Cu} / \mathrm{Cu}(111)$ pair potential is consistent with recent experimental $^{8,9}$ and DFT (Ref. 2) results. This potential is attractive from the first to the fifth neighbor distances- the strongest attraction is found at the first $(250 \mathrm{meV})$ and second $(150 \mathrm{meV})$ neighbors, after which the attraction fades to zero at the 6th-8th neighbor distances. $\mathrm{Cu}(111)$ also exhibits a repulsive ring with a magnitude of $30 \mathrm{meV}$ at the 9 th-11th neighbor distances $(\sim 12 \AA)$. As we elaborate below, this value is based on recent STM results, ${ }^{8,9}$ which measure the effect of the repulsion on the diffusion barrier for two adatoms to aggregate on $\mathrm{Cu}(111)$. Thus, the interaction profiles on these two surfaces are qualitatively similar.

In addition to pair interactions, trio, quarto, and higher order terms could exist in the Hamiltonian adopted here. For large adsorbate separations in the asymptotic regime, Hyldgaard and Einstein recently found that the electronic trio interaction can comprise as much as a quarter of the total interaction energy between three atoms on noble-metal fcc(111) surfaces. ${ }^{17}$ Though the effect of higher-order interactions is noticeable in principle, it is not important here and we neglect it. A detailed analysis of higher order terms will 


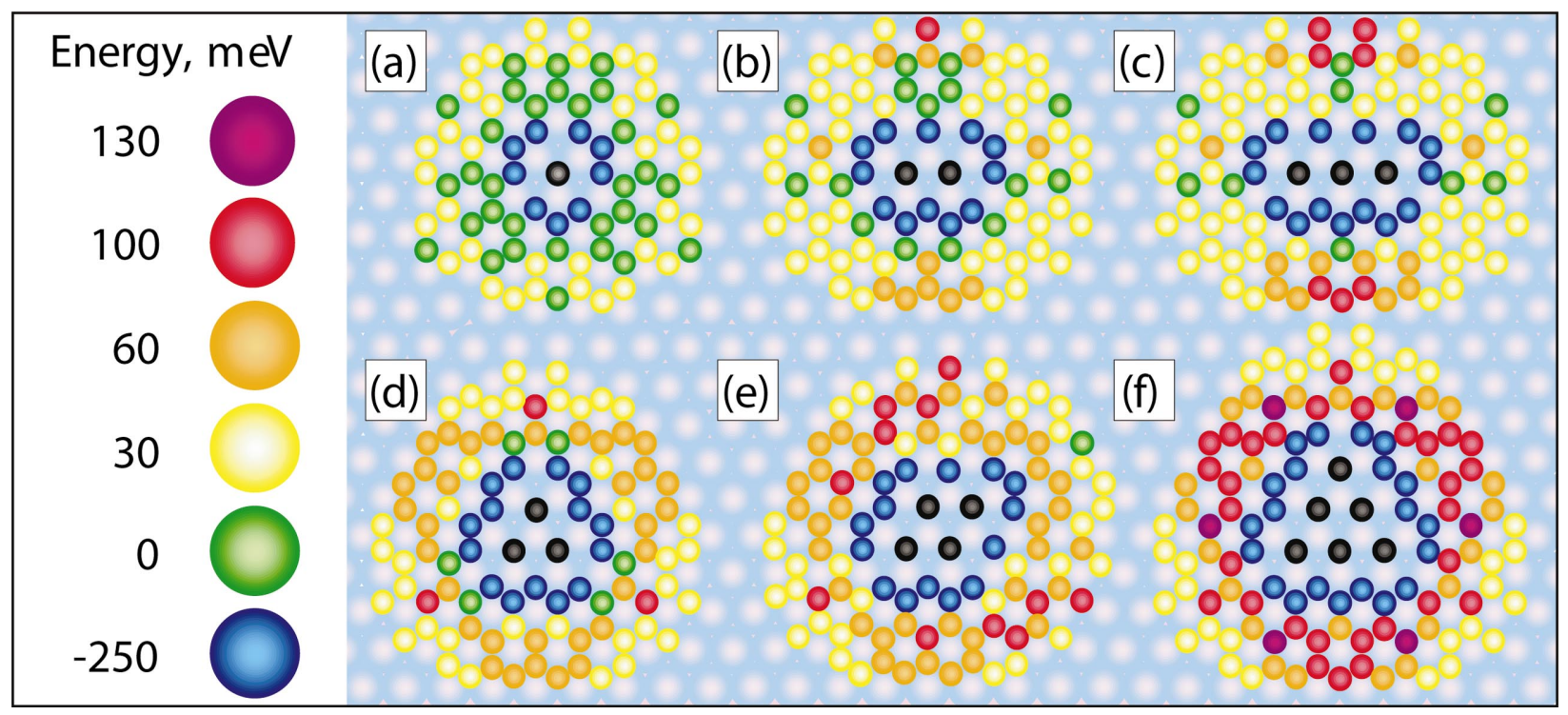

FIG. 1. (Color) Potential-energy maps for an adatom to approach another adatom (a) and small adsorbate islands (b) -(f) on strained $\mathrm{Ag}(111)$. Light circles in the light-blue background represent the centers of surface atoms and black circles represent adatoms.

be given elsewhere. ${ }^{16}$ Elastic interactions also play a role in these systems, but our DFT calculations for $\mathrm{Ag}(111)$ surfaces indicate that they are negligible. ${ }^{16}$

One consequence of "intermediate-range" interactions for the formation of nanostructures at surfaces is seen in the potential-energy map for an adatom to approach a small island. We obtain these maps by calculating the interaction energy (i.e., the sum of pair interactions) when an adatom is on various fcc and hep binding sites surrounding the island. The potential-energy map for a dimer is shown in Fig. 1(b). Overall, the repulsion surrounding a dimer is greater than that around a monomer. This repulsion is anisotropic and it is easier for an atom to approach the dimer at the ends than in the middle.

We observe a dependence of the potential-energy map on cluster shape for trimers. A linear trimer [Fig. 1(c)] has a similar potential-energy map to a dimer, although the repulsion parallel to the trimer axis is greater than that for the dimer. If the trimer is compact [Fig. 1(d)], the repulsion is isotropic and the low-energy (yellow in Fig. 1) paths that allow atoms to join the monomer, dimer, and the linear trimer no longer occur. Although linear trimers can grow more easily (via addition at the ends) than compact ones, they can also rearrange to the more stable compact form. Thus, the relative time scales for monomer addition and island rearrangement will determine the propensity for trimers to grow or persist over a fixed temperature range.

Progressing to larger clusters, we find that the tetramer exhibits the same trends as the trimer. When a tetramer is in the linear form, adatoms will add most easily to the ends. Further, the repulsion surrounding a compact tetramer [Fig. $1(\mathrm{e})]$ is stronger overall than that around the linear tetramer. Comparing the compact tetramer and trimer, we see that the potential-energy map around the tetramer is not as uniform because of its reduced symmetry. Nevertheless, a greater fraction of the sites around the tetramer periphery has repulsion in the 100-130-meV range (red in Fig. 1), making it more difficult for atoms to access the tetramer. We find similar results for a compact pentamer, which has an even greater fraction of periphery sites with energies in the 100-130-meV (red) range. The compact hexamer [Fig. 1(f)] is completely surrounded by sites with repulsion of $100 \mathrm{meV}$, or more, and the (orange) low-energy paths that we see for the compact trimer, tetramer, and pentamer have disappeared. Comparing the potential-energy maps surrounding all the small clusters, we find that it is increasingly difficult for an adatom to aggregate with a cluster as the cluster size increases. For a fixed size, compact clusters with high symmetry have the highest barriers preventing growth by monomer addition.

The trends for small clusters generally extend to larger clusters, although analysis is complicated by their large variety of possible shapes. Further, the number of trio (and higher-order) interactions begin to outnumber pairs for moderate-sized islands, although it is unclear whether these are significant. Nevertheless, as cluster sizes increase beyond $\sim 12$ atoms, interatomic distances in a reasonably compact cluster begin to exceed the range of the repulsive ring. At these distances, constructive interference of the atomic repulsive rings no longer occurs and short-range attraction can mitigate the intermediate-range repulsion. We note that irregularities on the perimeter of large islands create local potential-energy maps similar to those for small islands. Although our discussion has focused on the strained $\mathrm{Ag}(111)$ system, we find analogous results for $\mathrm{Cu} / \mathrm{Cu}(111)$.

To investigate the ramifications of these size- and shapedependent "island barriers" for the formation of nanostructures on surfaces, we conduct kMC simulations of thin-film growth. We use the general method of Fichthorn and Weinberg ${ }^{18}$ to simulate atom deposition with a rate of $F$ $(\mathrm{ML} / \mathrm{s})$ and adatom hopping from site $i$ to site $j$ with a rate $D_{i \rightarrow j}=D_{0} \exp \left(-E_{i \rightarrow j} / k_{B} T\right)$. The prefactor is set $D_{0}=10^{12}(9$ $\left.\times 10^{11}\right) \mathrm{s}^{-1}$ for the $\mathrm{Ag}(\mathrm{Cu})$ surface to be consistent with experimental ${ }^{9}$ and DFT (Ref. 19) results. The influence of adatom pair interactions on adsorbate diffusion (and, hence, 
island nucleation and growth) is included in the hopping barrier, which takes the form $E_{i \rightarrow j}=E_{b}^{0}+\frac{1}{2}\left(E_{j}-E_{i}\right) .{ }^{1}$ Here, $E_{b}^{0}$ is the barrier for an isolated adatom to hop on an otherwise bare surface-for strained $\operatorname{Ag}(111), E_{b}^{0}=52 \mathrm{meV}$ (Refs. 1 and 19) and for $\mathrm{Cu}(111), E_{b}^{0}=40 \mathrm{meV} \cdot{ }^{9} E_{i(j)}$ is the interaction energy (i.e., the sum of pair interactions) when an adatom is at site $i(j)$. Within this model, the "extra" barrier, above the bare-surface hopping barrier, for two adatoms to nucleate a dimer is $25 \mathrm{meV}$ for strained $\mathrm{Ag}(111)$ and $15 \mathrm{meV}$ for $\mathrm{Cu}(111)$. The value for $\mathrm{Cu}(111)$ is consistent with STM results. ${ }^{8,9}$

Several channels for island rearrangement occur in these systems, including adatom hopping at island edges and concerted, multiatom processes. ${ }^{20-23}$ These processes allow island rearrangement to occur over a wide range of growth conditions. Since we investigate small islands, generally containing less than ten atoms, equilibrium shapes can be attained with minimal rearrangement. Thus, it seems likely that small islands attain their compact, equilibrium distribution of shapes under typical experimental conditions. As discussed

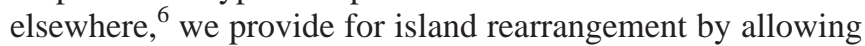
atoms with one or more neighbors to execute hops beyond the nearest-neighbor distance. These extra rate processes allow for the formation of compact islands with an equilibrium distribution of shapes. The influence of limited island rearrangement, if relevant, could be studied by including more realistic rate processes for island reshaping.

We simulate thin-film epitaxy at temperatures from 20 to $75 \mathrm{~K}$ and for fluxes of $F=0.1$ and $0.01 \mathrm{ML} / \mathrm{s}$. We quantify island-size distributions, obtained as averages over typically 100 runs, at a coverage of $\theta=0.07 \mathrm{ML}$. This coverage is approximately in the middle of the island nucleation regime prior to the steady-state, growth regime. Although we observe somewhat larger islands at coverages closer to the growth regime, our conclusions are not strongly coverage dependent.

At low temperatures, adatom aggregation is hindered by the repulsive ring and there are many isolated adatoms. It has been observed in analogous, low-temperature STM studies ${ }^{8,9}$ that pairs of atoms encounter each other many times without aggregating. As the temperature increases, adatoms aggregate with similar barriers to form dimers and linear trimers. When a linear trimer rearranges to the compact form, it attains a shape with a larger barrier against growth by monomer addition. Thus, we observe a large fraction of dimers and trimers, which comprise about $70 \%$ of the islands in the strained $\mathrm{Ag}(111)$ system at $35 \mathrm{~K}$ and $F=0.01$. To emphasize the sharpness of the island-size distribution at these conditions, we note that the mean island size is $\langle S\rangle=2.9$ and the standard deviation is $\sigma=1.4$. In the absence of a repulsive ring, with just nearest-neighbor attraction, we find $\langle S\rangle$ $=28.8$ and $\sigma=14.6$.

As the temperature increases, atoms are able to access the compact trimer. Since the compact trimer, tetramer, and pentamer have similar energy barriers surrounding them [cf., Figs. 1(d) and 1(e)], all of these structures can form and grow as the temperature increases. We see in Fig. 2 that at 50 $\mathrm{K}$, the fraction of dimers has dropped below $3 \%$ and most of the clusters are trimers, tetramers, and pentamers. By in-

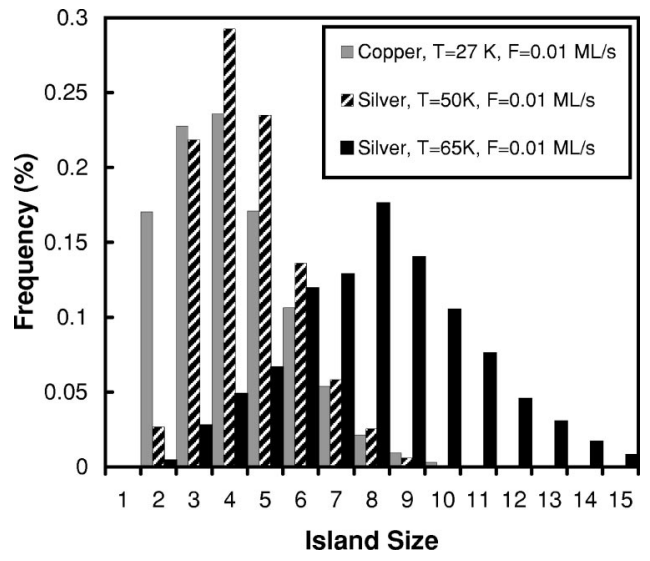

FIG. 2. Representative island-size distributions after 0.07 ML has been deposited.

creasing the temperature further, we facilitate the growth of hexamers, which are more resistant to monomer addition than the smaller clusters [cf., Fig. 1(f)]. As we see in Fig. 2, most of the islands are comprised of 6-11 atoms at $65 \mathrm{~K}$.

Because interactions are weaker for $\mathrm{Cu}(111)$, their effects are less pronounced. Although the repulsive ring prevents most atoms from nucleating dimers at $20 \mathrm{~K}$, aggregation becomes sufficiently facile at $25 \mathrm{~K}$ that over half the islands are dimers and trimers. At $27 \mathrm{~K}$, as we see in Fig. 2, most of the islands have $2-5$ atoms. This is also the case at $30 \mathrm{~K}$, although more than $30 \%$ of the islands have six or more atoms at this temperature.

The dependence of the average island size $\langle S\rangle$ on the temperature and deposition rate is summarized in Fig. 3. The strong temperature dependence of $\langle S\rangle$ for $\mathrm{Cu}(111)$ reflects its weak interactions. The size distributions are also the broadest for $\mathrm{Cu}(111)$. At low temperatures, the strong size dependence of the island barriers for strained $\operatorname{Ag}(111)$ effectively blocks growth via adatom diffusion when islands reach a certain size. Here, size distributions are very narrow, with $\sigma=1.2$ at

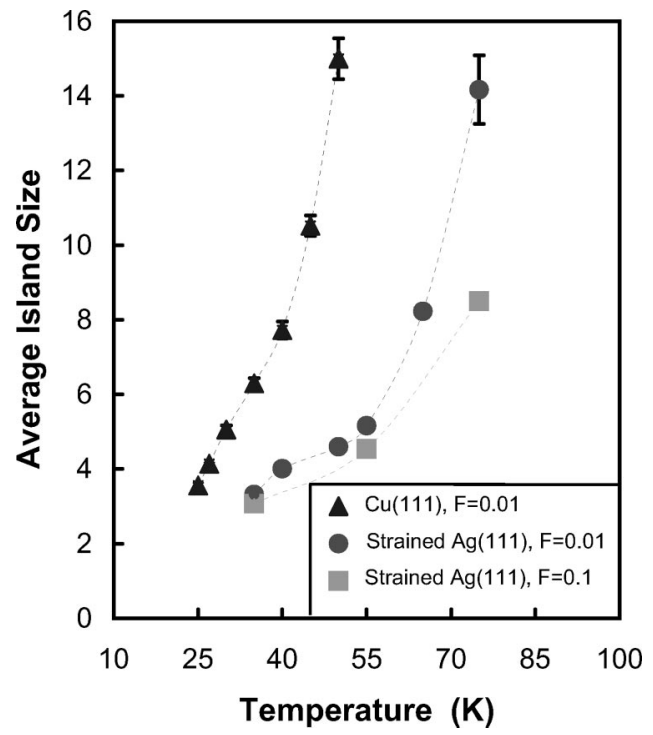

FIG. 3. Average island size as a function of temperature after 0.07 ML has been deposited. 
$35 \mathrm{~K}$ for $F=0.1 \mathrm{ML} / \mathrm{s}$, for example. As the temperature increases, island growth is mediated to an increasing extent by adatom diffusion and there is a stronger temperature dependence of $\langle S\rangle$ for strained $\operatorname{Ag}(111)$. The island-size distributions also broaden, with $\sigma=2.8$ at $75 \mathrm{~K}$ for $F=0.1 \mathrm{ML} / \mathrm{s}$.

Comparing $\langle S\rangle$ for strained $\operatorname{Ag}(111)$ at $F=0.1$ and 0.01 $\mathrm{ML} / \mathrm{s}$, we find larger $\langle S\rangle$ with $F=0.01$. When diffusion occurs more rapidly relative to deposition (as occurs for $F=0.01$ relative to $F=0.1$ ), larger islands can form. The size distributions are also broader for $F=0.01$ at higher temperatures.

In summary, intermediate-range, substrate-mediated interactions lead to the formation of repulsive barriers surround- ing small adsorbate islands. For small islands, these barriers increase with increasing island size and they depend on island shape. The size dependence of the island barriers leads to narrow island-size distributions, which can be tuned by manipulating growth conditions. We note that our DFT calculations indicate the interactions can be tuned by straining a surface during heteroepitaxy. ${ }^{1,16}$ These interactions and effects open new possibilities for creating well-defined nanostructures at surfaces.

This work was sponsored by the Alexander von Humboldt Foundation and the NSF (DMR-9617122, ECC-0085604, DGE-9987589).
${ }^{1}$ K.A. Fichthorn and M. Scheffler, Phys. Rev. Lett. 84, 5371 (2000).

${ }^{2}$ A. Bogicevic, S. Ovesson, P. Hyldgaard, B.I. Lundqvist, H. Brune, and D.R. Jennison, Phys. Rev. Lett. 85, 1910 (2000).

${ }^{3}$ P. Hyldgaard and M. Persson, J. Phys.: Condens. Matter 12, L13 (2000).

${ }^{4}$ S. Ovesson, A. Bogicevic, G. Wahnstrom, and B.I. Lundqvist, Phys. Rev. B 64, 125423 (2001).

${ }^{5}$ S. Ovesson, Phys. Rev. Lett. 88, 116102 (2002).

${ }^{6}$ K.A. Fichthorn, M.L. Merrick, and M. Scheffler, Appl. Phys. A: Mater. Sci. Process. 75, 17 (2002).

${ }^{7}$ J.A. Venables and H. Brune, Phys. Rev. B 66, 195404 (2002).

${ }^{8}$ J. Repp, M. Moresco, G. Meyer, K.H. Rieder, P. Hyldgaard, and H. Persson, Phys. Rev. Lett. 85, 2981 (2000).

${ }^{9}$ N. Knorr, H. Brune, M. Epple, A. Hirstein, M.A. Schneider, and K. Kern, Phys. Rev. B 65, 115420 (2002).

${ }^{10}$ K. Lau and W. Kohn, Surf. Sci. 65, 607 (1977); 75, 69 (1978).

${ }^{11}$ T.L. Einstein, Handbook of Surface Science, edited by W.N. Unertl (Elsevier, Amsterdam, 1996), Vol. 1, p. 577.

${ }^{12}$ H. Brune, K. Bromann, H. Roeder, K. Kern, J. Jacobsen, P. Stoltze, K. Jacobsen, and J. Norskov, Phys. Rev. B 52, R14 380
(1995).

${ }^{13}$ J.V. Barth, H. Brune, B. Fischer, J. Weckesser, and K. Kern, Phys. Rev. Lett. 84, 1732 (2000).

${ }^{14}$ M. Bockstedte, A. Kley, J. Neugebauer, and M. Scheffler, Comput. Phys. Commun. 107, 187 (1997).

${ }^{15}$ J.P. Perdew, K. Burke, and M. Ernzerhof, Phys. Rev. Lett. 77, 3865 (1996).

${ }^{16}$ W. Luo, K.A. Fichthorn, and M. Scheffler (to be published).

${ }^{17}$ P. Hyldgaard and T.L. Einstein, Europhys. Lett. 59, 265 (2002).

${ }^{18}$ K.A. Fichthorn and W.H. Weinberg, J. Chem. Phys. 95, 1090 (1991).

${ }^{19}$ C. Ratsch and M. Scheffler, Phys. Rev. B 58, 13163 (1998).

${ }^{20}$ P. Ruggerone, C. Ratsch, and M. Scheffler, "Growth and Properties of Ultrathin Epitaxial Layers," The Chemical Physics of Solid Surfaces, Vol. 8, edited by D.A. King and D.P. Woodruff (Elsevier, Amsterdam, 1997) p. 490.

${ }^{21}$ S. Ovesson, A. Bogicevic, and B.I. Lundqvist, Phys. Rev. Lett. 83, 2608 (1999).

${ }^{22}$ B.-D. Yu and M. Scheffler, Phys. Rev. B 55, 13916 (1997).

${ }^{23}$ R.A. Miron and K.A. Fichthorn, J. Chem. Phys. 115, 8742 (2001). 